Title : will be set by the publisher

Editors : will be set by the publisher

EAS Publications Series, Vol. ?, 2018

\title{
ASTROMETRY AND EXOPLANETS: THE GAIA ERA, AND BEYOND
}

\author{
A. Sozzetti ${ }^{1}$
}

\begin{abstract}
The wealth of information in the Gaia catalogue of exoplanets will constitute a fundamental contribution to several hot topics of the astrophysics of planetary systems. I briefly review the potential impact of Gaia micro-arsec astrometry in several areas of exoplanet science, discuss what key follow-up observations might be required as a complement to Gaia data, and shed some light on the role of next generation astrometric facilities in the arena of planetary systems.
\end{abstract}

\section{Introduction}

The study of exoplanets orbiting solar-type stars has emerged in the last decade to be one of the most exciting new areas of astronomy and planetary science. Since 1995, almost 500 planets outside the solar system have been discovered, mostly by the radial-velocity method (a technique that can only put lower limits on the mass of any detected companion). Collectively, they span a huge range of masses and orbital distances, are commonly found in multiple systems (the record-holder ' being HD 10180 with its 7-planet system), have been detected around star of varied spectral types and evolutionary stages, and have led to major revisions in our ideas of how and where planets form and what their structure is. The majority of these planets are gas giants, but more than 70 are of Neptune mass and lower. Ninety-seven (four of which in multiple-planet systems) are known to transit across the disk of their primary star, and it is for these that we have physical parameters such as radius and mass. A subset of the close-in transiting giants has now been detected directly at secondary eclipse and at various orbital phases by the Spitzer Infrared Space Telescope and the Hubble Space Telescope, providing the first measurements of extrasolar planetary atmospheres and compositions. To interpret these data, theorists have developed models for planet formation, orbital interaction and dynamics, evaporation due to stellar irradiation, atmospheric

\footnotetext{
1 INAF - Osservatorio Astronomico di Torino, Strada Osservatorio, 20 - 10025 Pino Torinese Italy 
Title : will be set by the publisher

circulation and global heat transport, atmospheric structure and spectra, phase light curves, the equations of state of their interiors, molecular chemistry, radius evolution, and tidal effects, to name only a few topics.

Their emerging properties in the near future may be put on much more solid statistical grounds, thanks to an going and soon to become larger flow of observational data collected with a variety of techniques which help answer some of the most outstanding questions what some refer to now as the science of "comparative exoplanetology". Among the vast array of techniques for planet detection and characterization, the potential of high-precision astrometric measurements is still mostly unexplored. After decades of 'blunders' (e.g., Sozzetti 2010, and references therein), micro-arcsecond ( $\mu$ as) astrometry is now coming of age, which will allow this technique to obtain in perspective the same successes of the Doppler method, for which the improvement from the $\mathrm{km} \mathrm{s}^{-1}$ to the $\mathrm{m} \mathrm{s}^{-1}$ precision opened the doors for ground-breaking results in exoplanetary science. I briefly review here what promises the next decade holds for astrometry and exoplanets.

\section{Gaia and extrasolar planets}

Gaia's ambitious science case (e.g., Perryman et al. 2001), wishing to address breakthrough problems in Milky Way astronomy, great impact is the astrophysics of planetary systems (e.g., Casertano et al. 2008), in particular when seen as a complement to other techniques for planet detection and characterization (e.g., Sozzetti 2010). Given the characteristics of Gaia's magnitude-limited survey (see e.g. the reviews by Prusti and Charvet, this volume), and the non-competitiveness of its spectroscopic and photometric capabilities with facilities devoted to highprecision radial-velocity measurements (e.g., Pepe \& Lovis 2008) or transit photometry (e.g., Sozzetti et al. 2010), the mission's potential contribution to exoplanets science must be purely gauged in terms of its astrometric capabilities.

\subsection{The Gaia Double-Blind Tests Campaign}

In the large-scale, double-blind test campaign carried out to estimate the potential of Gaia for detecting and measuring planetary systems, Casertano et al. (2008) showed that, considering bright stars $(V<13)$ uniformly observable with the best-achievable single-measurement astrometric precision $\left(\sigma_{\psi} \simeq 10-15 \mu\right.$ as), Gaia could discover and measure massive giant planets $\left(M_{\mathrm{p}} \geq 2-3 M_{\mathrm{J}}\right)$ with $1<a<4$ AU orbiting solar-type stars as far as the nearest star-forming regions, as well as explore the domain of Saturn-mass planets with similar orbital semi-major axes around late-type stars within 30-40 pc (see Figure 1). These results can be used to infer the number of planets of given mass and orbital separation that can be detected and measured by Gaia, using Galaxy models and the current knowledge of exoplanet frequencies. By inspection of the two sub-tables in Table 1, one then finds that Gaia's main strength will be its ability to accurately measure orbits and masses for thousands of giant planets, and to perform coplanarity measurements for a few hundred multiple systems with favorable configurations. 


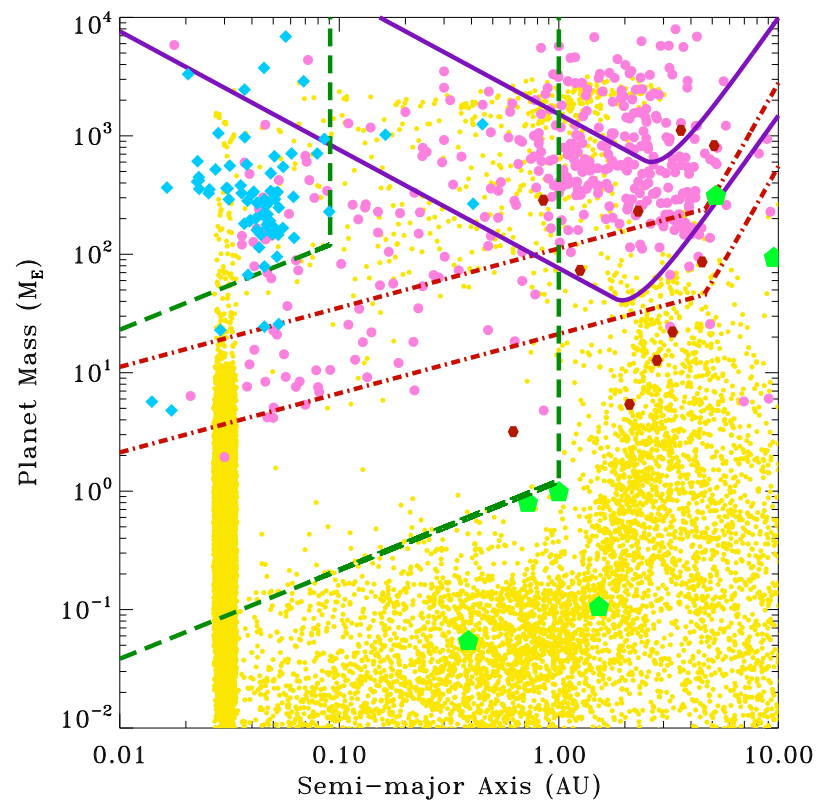

Fig. 1. Gaia exoplanets discovery space (blue curves) compared to that of Doppler (red lines) and transit (green curves) techniques. Detectability curves are defined on the basis of a 3- $\sigma$ criterion for signal detection (see Sozzetti 2010 for details). The upper and lower blue solid curves are for Gaia astrometry with $\sigma_{\mathrm{A}}=10 \mu \mathrm{as}$, assuming a $1-M_{\odot}$ primary at $200 \mathrm{pc}$ and a $0.4-M_{\odot} \mathrm{M}$ dwarf at $25 \mathrm{pc}$, respectively, and survey duration set to 5 yr. The pink filled circles indicate the inventory of Doppler-detected exoplanets as of May 2010. Transiting systems are shown as light-blue filled diamonds. Red hexagons are planets detected by microlensing. Solar System planets are also shown (large green pentagons). The small yellow dots represent a theoretical distribution of masses and final orbital semi-major axes (Ida \& Lin 2008).

Finally, a word of caution is in order on the face of the possible degradations in the expected Gaia astrometric precision on bright stars $(V<13)$. As the refinement of the overall Gaia error budget progresses, including a better understanding of some effects which can potentially affect centroiding as well as systematic uncertainties to a significant degree, such as the charge transfer inefficiency effect due to solar radiation bombardment of Gaia CCDs at L2 (see e.g. Prod'homme and Pasquier, this volume), a worsening of Gaia astrometric performance might impact more or less seriously the Gaia science case for exoplanets. Casertano et al. (2008) have shown how the number of useful stars, and of detectable and measurable single- and multiple-planet systems would decrease as $\sigma_{\psi}$ increases (assuming that the number of objects scales with the cube of the radius, in pc, of a sphere centered around the Sun). In the context of that exercise, a factor 2 degradation in astrometric precision would impact most of Gaia exoplanet science case. Until the 
Title : will be set by the publisher

true Gaia performance on real data will be known, it will be necessary to closely follow further developments in the understanding of the technical specifications of Gaia and its instruments, and of its observation and data analysis process, in order to revisit these issues as needed in the future.

\begin{tabular}{|c|c|c|c|c|c|}
\hline $\begin{array}{c}\Delta d \\
(\mathrm{pc})\end{array}$ & $N_{\star}$ & $\begin{array}{c}\Delta a \\
(\mathrm{AU})\end{array}$ & $\begin{array}{c}\Delta M_{p} \\
\left(M_{J}\right)\end{array}$ & $N_{\mathrm{d}}$ & $N_{\mathrm{m}}$ \\
\hline $0-50$ & $1 \times 10^{4}$ & $1.0-4.0$ & $1.0-13.0$ & 1400 & 700 \\
\hline $50-100$ & $5 \times 10^{4}$ & $1.0-4.0$ & $1.5-13.0$ & 2500 & 1750 \\
\hline $100-150$ & $1 \times 10^{5}$ & $1.5-3.8$ & $2.0-13.0$ & 2600 & 1300 \\
\hline $150-200$ & $3 \times 10^{5}$ & $1.4-3.4$ & $3.0-13.0$ & 2150 & 1050 \\
\hline
\end{tabular}

(a)

\begin{tabular}{|l|c|}
\hline Case & N. of systems \\
\hline Detection & $\sim 1000$ \\
\hline $\begin{array}{l}\text { Orbits and masses to } \\
<15-20 \% \text { accuracy }\end{array}$ & $\sim 400-500$ \\
\hline $\begin{array}{l}\text { Successful } \\
\text { coplanarity tests }\end{array}$ & $\sim 150$ \\
\hline
\end{tabular}

(b)

Table 1. Left: Number of giant planets of given ranges of mass $\left(\Delta M_{p}\right)$ and orbital separation $(\Delta a)$ that could be detected $\left(N_{d}\right)$ and measured $\left(N_{m}\right)$ by Gaia, as a function of increasing distance $(\Delta d)$ and stellar sample $\left(N_{\star}\right)$. Right: Number of planetary systems that Gaia could potentially detect, measure, and for which coplanarity tests could be carried out successfully. See Casertano et al. (2008) for details.

\subsection{Astrometric Modeling of Planetary Systems}

The problem of the correct determination of the astrometric orbits of planetary systems using Gaia data (highly non-linear orbital fitting procedures, large numbers of model parameters) will present many difficulties. For example, it will be necessary to assess the relative robustness and reliability of different procedures for orbital fits, with a detailed understanding of the statistical properties of the uncertainties associated with the model parameters. For multiple systems, a trade-off will have to be found between accuracy in the determination of the mutual inclination angles between pairs of planetary orbits, single-measurement precision and redundancy in the number of observations with respect to the number of estimated model parameters. It will be challenging to correctly identify signals with amplitude close to the measurement uncertainties, particularly in the presence of larger signals induced by other companions and/or sources of astrophysical noise of comparable magnitude. Finally, for systems where dynamical interactions are important (a situation experienced already by Doppler surveys), fully dynamical fits involving an n-body code might have to be used to properly model the Gaia astrometric data and to ensure the dynamical stability of the solution (see Sozzetti 2005). All the above issues could significantly impact Gaias planet detection and characterization capabilities. For these reasons, a Development Unit (DU), within the pipeline of Coordination Unit 4 (object processing) of the Gaia Data Processing and Analysis Consortium, has been specifically devoted to the modelling of the astrometric signals produced by planetary systems. The DU is composed of several tasks, which implement multiple robust procedures for (single and multiple) astrometric orbit fitting (such as Markov Chain Monte Carlo algorithms) and the determination of the degree of dynamical stability of multi-planet systems. 


\subsection{The Gaia Legacy}

Gaia's main contribution to exoplanet science will be its unbiased census of planetary systems orbiting hundreds of thousands nearby $(d<200 \mathrm{pc})$, relatively bright $(V \leq 13)$ stars across all spectral types, screened with constant astrometric sensitivity. As a result, the actual impact of Gaia measurements in exoplanets science is broad, and rather structured. The Gaia data have the potential to: a) significantly refine our understanding of the statistical properties of extrasolar planets; b) help crucially test theoretical models of gas giant planet formation and migration; c) achieve key improvements in our comprehension of important aspects of the formation and dynamical evolution of multiple-planet systems; d) aid in the understanding of direct detections of giant extrasolar planets; e) provide important supplementary data for the optimization of the target selection for future observatories aiming at the direct detection and spectral characterization of habitable terrestrial planets. Finally, ongoing studies (Sozzetti et al., in preparation) are now focusing on the detailed understanding of the planet discovery potential of Gaia as far as low-mass and post-main-sequence stars and are concerned.

\subsection{Synergies Between Gaia and Other Programs}

The broad range of applications to exoplanets science is such that Gaia data can be seen as an ideal complement to (and synergy with) many ongoing and future observing programs devoted to the indirect and direct detection and characterization of planetary systems, both from the ground and in space. Gaia will contribute critically, for example, to the definition of input catalogues for proposed quasi-allsky photometric transit surveys (PLATO); it will inform direct imaging surveys (e.g., SPHERE/VLT, EPICS/E-ELT) and spectroscopic characterization projects about the epoch and location of maximum brightness of (primarily non transit-

ing) exoplanets, in order to estimate their optimal visibility, and will help in the modeling and interpretation of giant planets' phase functions and light curves.

Another critical aspect will concern the large effort in terms of ground-based follow-up activities to improve the characterization of astrometrically detected systems (and possibly those found transiting). For example, high-precision radialvelocity campaigns (both at visible and infrared wavelengths) will be a necessary complement, with the three-folded aim of improving the phase sampling of the astrometric orbits found by Gaia, extending the time baseline of the observations (to put stringent constraints on or actually characterize long-period companions), and search for additional, low-mass and/or short-period components which might have been missed by Gaia due to lack of sensitivity.

\section{High-Precision Astrometry for Exoplanets: Beyond Gaia}

In addition to Gaia, there are several astrometric projects devoted to exoplanet detection and characterization (e.g, Sozzetti 2010; Malbet et al. 2010) both from 
Title : will be set by the publisher

the ground and in space, which are either ongoing, about to start, or under development/study. They vary in accuracy level and number of potential targets.

Ground-based relative astrometry programs with large telescopes and adaptive optics (VLT/FORS2, CAPSCam, STEPSS) are focusing on relatively small numbers (a few tens to one hundred) of very faint targets (late M, L, and T dwarfs) in dense stellar fields. The expected long-term precision of these observatories will not exceeed $0.1-1$ mas. The performance of instrumentation for coronagraphic astrometry has been studied (e.g., Digby et al. 2006) and found not to be competitive in term of achievable precision, for the time being. Ground-based dual-star interferometry projects (VLTI/PRIMA, ASTRA) are designed to perform narrowangle interferometric astrometry of a very bright target and one moderately faint reference star separated by up to $1^{\prime}$ with expected accuracies better than $100 \mu$ as. Also in this case, the target lists will be composed by typically 100 stars.

In space, HST/FGS can still guarantee an astrometric precision of $0.3-0.5$ mas (exceeding that of Hipparcos), for the improved characterization of selected, suitable systems known to host massive planets from Doppler observations. The long-term ( $\sim 20$ years) SIM/SIM-Lite optical interferometry project, capable of narrow-field astrometry with 1- $\mu$ as precision, has recently not been recommended for funding by NASA's Decadal Survey. No space-borne astrometric facility is now foreseen to exceed Gaia's astrometric performance during or after the end of Gaia operational phase. Studies of the projected performance of high-precision narrow-angle coronagraphic astrometry in space are still at the infancy status.

\section{Conclusions}

The Gaia mission is now set to establish the European leadership in high-precision astrometry for the next decade. The largest compilation of high-accuracy astrometric orbits of giant planets, unbiased across all spectral types up to $d \simeq 200$

pc, will allow Gaia to crucially contribute to several aspects of planetary systems astrophysics (formation theories, dynamical evolution), in combination with present-day and future extrasolar planet search programs.

\section{References}

Casertano, S., Lattanzi, M. G., Sozzetti, A., et al. 2008, A\&A, 482, 699

Digby, A. P., Hinkley, S., Oppenheimer, B. R., et al. 2006, ApJ, 650, 484

Ida, S., \& Lin, D. N. C. 2008, ApJ, 673, 487

Malbet, F., Sozzetti, A., Lazorenko, P. et al. 2010, ASP Conf. Ser., 430, 84

Pepe, F., \& Lovis, C. 2008, Physica Scripta, 130, 014007

Perryman, M. A. C., et al. 2001, A\&A, 369, 339

Sozzetti, A. 2005, PASP, 117, 1021

Sozzetti, A. 2010, EAS Publication Series, 42, 55

Sozzetti, A., Afonso, C., Alonso, R., et al. 2010, ASP Conf. Ser., 430, 45 